Projets

de paysage

\section{Projets de paysage}

Revue scientifique sur la conception et l'aménagement de l'espace

\section{3 | 2015}

Biodiversité et paysage

\title{
Pour de nouvelles rencontres en Pays de Caux
}

Enrichir le vivre ensemble à partir des espaces de transition. Apports de l'atelier consacré aux lisières aux alentours du Havre

For a New Community Life in the Pays de Caux Region - Enhancing Community Life Through Transition Spaces. Contributions from the Workshop on the Fringe Areas of the Outskirts of Le Havre

\section{Boris Menguy}

\section{(2) OpenEdition}

\section{Journals}

\section{Édition électronique}

URL : http://journals.openedition.org/paysage/9961

DOI : 10.4000/paysage.9961

ISSN : 1969-6124

\section{Éditeur :}

École nationale supérieure du paysage de Versailles-Marseille, Institut national des sciences appliquées Centre Val de Loire - École de la nature et du paysage, École nationale supérieure d'architecture et de paysage de Bordeaux, École nationale supérieure d'architecture et de paysage de Lille, Agrocampus Angers

\section{Référence électronique}

Boris Menguy, "Pour de nouvelles rencontres en Pays de Caux », Projets de paysage [En ligne], 13 |

2015, mis en ligne le 31 décembre 2015, consulté le 04 septembre 2020. URL : http://

journals.openedition.org/paysage/9961 ; DOI : https://doi.org/10.4000/paysage.9961

Ce document a été généré automatiquement le 4 septembre 2020

Projets de paysage 


\section{Pour de nouvelles rencontres en Pays de Caux}

Enrichir le vivre ensemble à partir des espaces de transition. Apports de l'atelier consacré aux lisières aux alentours du Havre

For a New Community Life in the Pays de Caux Region - Enhancing Community

Life Through Transition Spaces. Contributions from the Workshop on the Fringe

Areas of the Outskirts of Le Havre

Boris Menguy

Dans «Mon village en l'an 2000 », Charles-Henri Tachon décrit l'évolution de son village viticole en Bourgogne (Tachon, 1997). L'analyse fine des processus de transformation montre comment la cohérence du paysage agricole de Mercurey se perd dans une addition d'interventions techniques juxtaposées s'affranchissant des qualités $\mathrm{du}$ site où elles se déploient. Sans que nul n'ait vraiment décidé de la résultante ni imaginé la forme du village et de ses abords à terme, les interventions se succèdent et façonnent un nouveau village en rupture franche avec son passé. Cette approche à l'échelle d'un village encore épargné par l'influence d'une quelconque agglomération constitue une précieuse pierre à l'édifice de la compréhension d'un phénomène décuplé par la proximité des pôles urbains. Avec son titre oxymore, l'ouvrage Campagnes urbaines nous interpellait déjà sur une nouvelle manière d'appréhender les territoires périurbains et d'y décrypter le rôle précieux de certains espaces agricoles (Donadieu, 1998). Puis en 2004, David Mangin propose un éclairage nouveau, en considérant les territoires du périurbain comme des espaces structurés par la grande distribution et la motorisation (Mangin, 2004).

2 Alors même qu'ils accueillent de plus en plus d'activités et d'habitants, ces pans de territoire semblent échapper à tout contrôle et notamment à celui de la planification. C'est dans ce cadre qu'en 2005, Bernard Reichen et Alfred Peter expérimentent leur concept d'inversion du regard lors de l'élaboration du Scot de l'agglomération de Montpellier, document d'urbanisme qui fait date (Reichen et Peter, 2006). Les concepteurs proposent de «faire parler la géographie » en considérant que les espaces 
agricoles et de nature doivent devenir des « acteurs du projet ", c'est-à-dire en capacité à structurer et à donner des limites qualitatives pour guider les dynamiques de développement. Parallèlement, depuis plus de 25 ans en Allemagne, des pistes intéressantes de restructuration de territoires aussi peu qualifiés que la plupart de nos franges urbaines sont révélées par les démarches partenariales des Internationale Bauausstellung (IBA) (Beltrando et al., 2009). La voie du projet est privilégiée pour revitaliser ou accélérer les transitions de territoire. L'IBA d'Hambourg (2006-2013), portée par une forte implication habitante, organise le désenclavement d'une vaste île urbanisée de l'Elbe proche du centre. Cet urbanisme de projet participatif et durable est vecteur d'espaces plus qualitatifs et plus imaginatifs. Il contribue à la réappropriation de sites en déshérence, en difficulté économique et sociale.

3 En septembre 2013, la $34^{\mathrm{e}}$ rencontre des agences d'urbanisme, "Les campagnes urbaines, un avenir à bâtir", se saisit plus "frontalement» de la question du périurbain (Urbanisme, 2013). Les participants se penchent sur ces espaces longtemps dénigrés par les professionnels pour tenter d'y porter un regard constructif, d'admettre qu'ils concernent de près ou le loin une grande part de nos concitoyens et qu'il faut en imaginer des évolutions positives, un début de structuration.

4 La démarche d'atelier (2014) abordée dans cet article s'inscrit dans la filiation des approches et des expérimentations précitées. Elle bénéficie d'un cadre déjà en place : une charte paysagère et environnementale signée par les élus en 2011 et un Scot approuvé en 2012. Afin d'aller plus loin, cet atelier a pour objectif d'imaginer collectivement, à travers une dynamique de projet de paysage participatif axée sur les interfaces, des manières d'aboutir à des évolutions valorisantes (perception et usages) et structurantes du territoire. Il mobilise intentionnellement les ingrédients et les acteurs «habituels» de la transformation des territoires, en particulier périurbains (agriculture de proximité, gestion des eaux pluviales, création de cheminements...), dans un registre plus qualitatif et multifonctionnel.

\section{Métropolisation et attractivité}


Figure 1. L'embouchure de la Seine, une place géostratégique incarnée par le port du Havre et son ouverture vers les échanges maritimes internationaux

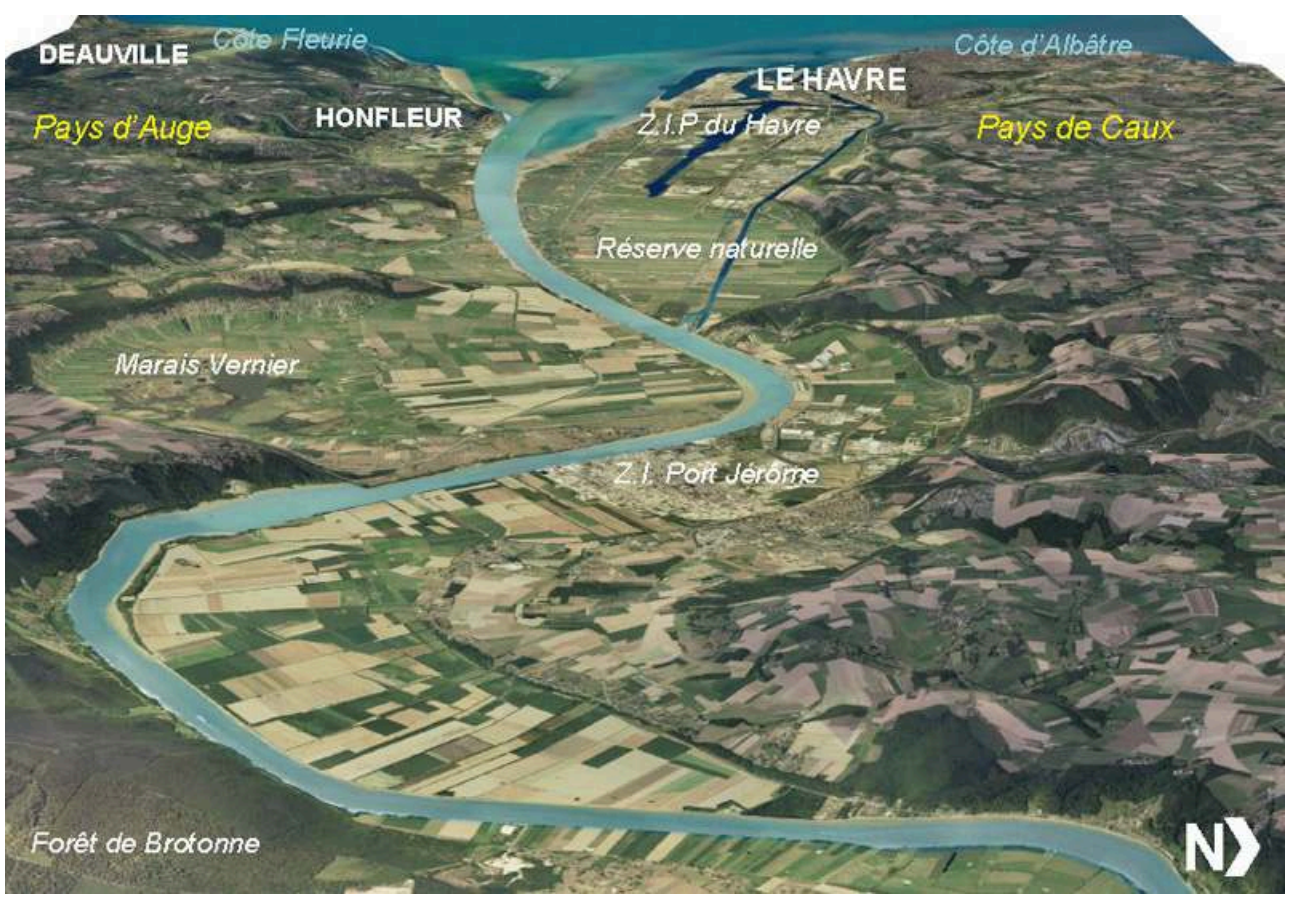

Une véritable mosaïque paysagère compose la plaine alluviale et témoigne de la diversité de ses usages.

Entre Manche et Seine, le territoire de la Pointe de Caux, emmené par le port du Havre, bénéficie d'une dynamique métropolitaine aux ressources importantes et fortement liée à une place géostratégique toujours plus affirmée. Si la somme de ces atouts constitue le socle d'un territoire qui comptera de plus en plus dans les années à venir, son attractivité n'est pas encore au rendez-vous. Le territoire manque de cohésion et d'une attention plus importante à son image et à son cadre de vie. De plus, malgré l'obtention d'un label Unesco pour le processus de la reconstruction du Havre et deux décennies intenses de projets urbains particulièrement visibles et qualifiants pour la place havraise (tramway, grand stade, entrée de ville, jardins suspendus, renouvellement urbain et portuaire...), la ville et l'espace du Scot continuent de perdre de la population.

Depuis 2009, portée par la logique du Grand Paris proposée par l'équipe d'Antoine Grumbach ${ }^{1}$, l'Agence d'urbanisme de la région du Havre et de l'Estuaire de la Seine $(\mathrm{AURH})^{2}$ contribue à un vaste projet de métropolisation à l'échelle de la Vallée de la Seine, appuyé sur le modèle de la "ville territoire». Les fondements de cette organisation reposent sur des alternances fonctionnelles (ville, agriculture, nature, industrie) et une logique d'imbrication, voire d'hybridation plus profitable des espaces. Cette «nouvelle architecture territoriale » se voit décliner à l'échelle des Scot, entrée privilégiée pour asseoir à des échelles pertinentes de telles approches. Dynamiques industrialo-portuaire, environnementale, touristique, patrimoniale, agricole, urbaine..., inéluctablement le territoire est amené à penser ses secteurs de développement ou de renouvellement selon des enjeux qui peuvent apparaître parfois contradictoires. Aussi, dans le squelette tenant ce vaste espace, la puissante structure géographique représente-t-elle un cadre précieux : falaises de la Côte d'Albâtre, estuaire de la Seine, plateau de Caux, vallées et valleuses. Mais au-delà, il reste à définir des espaces de 
transition évitant des confrontations malheureuses, des extensions urbaines sans limites, et offrant des lieux intermédiaires valorisants et ressourçants. Comment prétendre à une forme de distinction sans maîtriser le développement des « entredeux », comment organiser des cohabitations inédites, comment offrir un cadre de vie propre à stimuler l'attractivité du territoire? Autant de préoccupations pour lesquelles le travail sur les interfaces pourrait apporter des réponses intéressantes: c'est l'hypothèse que nous avions formulé en amont de l'atelier.

7 En effet, après avoir mené depuis 2009 un travail approfondi d'étude et de propositions sur des approches paysagères, environnementales et agricoles du territoire ${ }^{3}$ (Menguy et Guibert, 2014), l'AURH a proposé aux élus de définir ce nouvel axe de travail comme une déclinaison de la charte paysagère et environnementale ${ }^{4} \mathrm{du}$ Scot. Nous avions préalablement démontré que c'était dans les espaces d'interface qui sont autant de lieux de transition, de basculement et de limites que se jouaient les principales questions de qualité paysagère, les meilleures perspectives d'amélioration de la biodiversité et le renforcement des capacités de résilience du territoire. Nous avions l'intime conviction que c'était aussi dans ces espaces que se faisait et se défaisait l'image de ce territoire, que son évolution était la plus lisible. C'est donc sur cette thématique que le travail exploratoire avec l'École nationale supérieure du paysage (ENSP) Versailles-Marseille s'est concentré pendant cinq mois entre octobre 2013 et mars 2014 afin d'élaborer par la voie du projet des pistes nouvelles de valorisation.

\section{Comment aborder ces entre-deux qui posent question?}

8 La notion de «lisière » interroge la question de la limite mais également celle de la perméabilité des éléments du paysage. Le « contenu » de cet entre-deux constitue un des points clés. Cette zone d'échange et de partage représente souvent une zone sensible, soumise à de fortes pressions. Par sa nature, cet espace d'une grande richesse potentielle peut devenir aussi le reflet de conflits et d'une absence de projet. Zones d'interface, les lisières sont en effet des espaces que divers acteurs convoitent et prospectent dans des directions parfois opposées. Il est ainsi nécessaire de trouver les bons équilibres afin de passer de « confrontations malheureuses » à des « cohabitations inédites et fertiles ». Cela nous amène à appréhender l'épaisseur de ces "lisières ", leurs rôles et leurs statuts.

Par souci d'intelligibilité et de communication, nous avons tenté dans ce travail de ramener à trois situations théoriques la grande diversité de cas observés sur le terrain. 


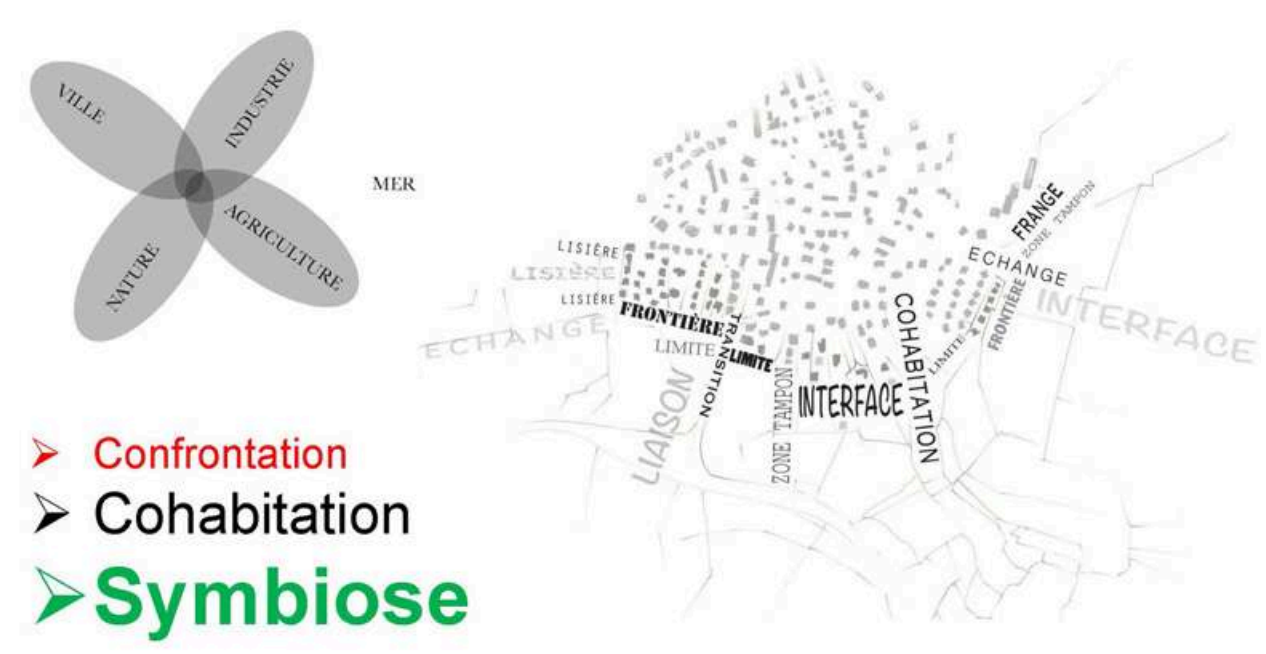

Par la diversité des usages du sol et des situations constatées sur le territoire, les interfaces apparaissent nécessairement multiples. Les rapports qui en découlent n'engendrent pas les mêmes qualités spatiales, nous en avons recensé trois principales. Les situations de symbiose actuelles représentent une source d'inspiration et un état souhaitable pour l'ensemble des interfaces à terme.

La confrontation correspond à un état insatisfaisant dans son maintien car il entérine une forme conflictuelle dans l'espace. Elle est bien illustrée par le cas très classique du lotissement ou du pavillon qui semble « débarquer » dans un espace agricole. C'est bien souvent l'opportunisme foncier qui engendre le rapprochement de deux usages du sol répondant à des logiques très différentes et susceptibles de se heurter. Dans cette configuration, l'absence de dispositif de transition est flagrante.

Dans la cohabitation, la limite est claire mais n'offre pas pour autant de qualité ni d'usage particulier, les interactions sont faibles. Cette forme de juxtaposition n'est $a$ priori pas conflictuelle.

11 Nous avons nommé symbiose le troisième cas. Généralement, entre deux usages du sol marquants vient s'inscrire un espace de partage qui bénéficie au territoire et aux deux espaces pour lesquels il assure un rôle d'interface avantageux. C'est la situation que nous souhaitions naturellement voir se développer et que nous avions pu identifier sur certains secteurs privilégiés du territoire.

L'atelier s'est placé dans le prolongement des divers travaux élaborés dans le cadre du Scot, il a ainsi intégré un état de connaissance avancé de la Pointe de Caux. Une approche fine et sensible du territoire a été rendue nécessaire afin que les étudiants puissent en "saisir » les spécificités mais nous avons réussi à économiser un temps précieux sur les aspects diagnostics et enjeux, déjà bien maîtrisés, pour nous concentrer sur une démarche de type work in progress. Cinq grandes entités ont été définies, où la question des lisières se manifeste différemment. Les espaces industrialoportuaires et les secteurs urbains denses ayant déjà bénéficié d'approches dédiées ${ }^{5}$ ont été volontairement écartés de la réflexion. Le principe du laboratoire d'idées a été retenu pour faciliter l'émergence d'exemples d'interventions concrètes et transposables. La réflexion s'est construite à partir des problématiques de sites pilotes ayant fait l'objet de choix partagés avec un comité technique et un comité de pilotage. Démultipliées, ces propositions locales, qui répondent aux enjeux spatialisés du Scot, 
s'intègrent dans des propositions d'ensemble à l'échelle de chaque entité paysagère et peuvent alors avoir une portée à l'échelle du Scot.

Figure 3. Carte des sites pilotes. Extrait du travail de l'atelier

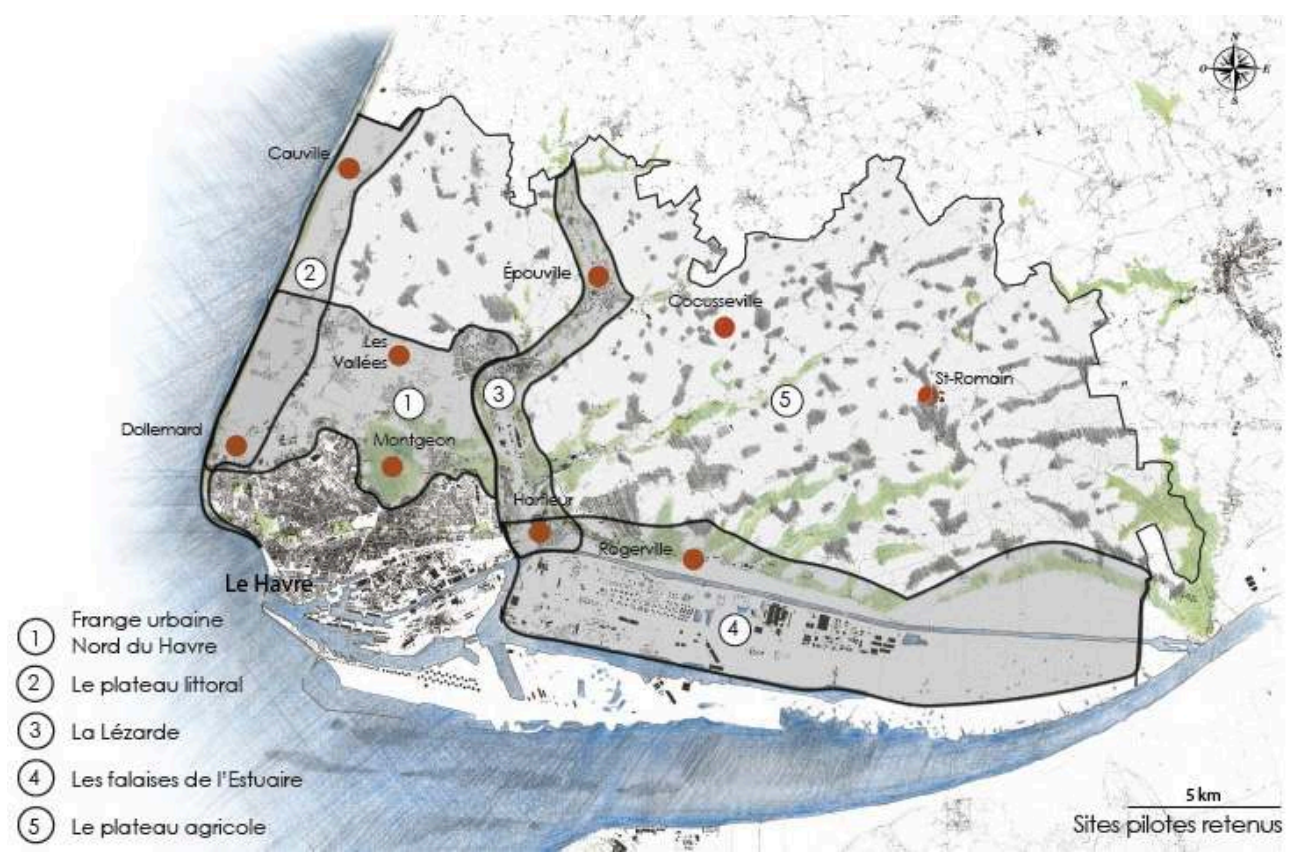

Quelques exemples locaux de lisières, à nos yeux porteurs de la philosophie de l'espace de partage, avaient déjà nourri le référentiel de la charte paysagère et environnementale du Scot. Les cas les plus démonstratifs montrent bien l'intérêt d'agir et la possibilité de s'inspirer de structures paysagères ${ }^{6}$ porteuses de sens. Nous avions eu l'occasion de présenter certains cas en amont de l'atelier et ils ont permis d'illustrer l'intention de ce dernier. Le projet de la plage du Havre conçu par Alexandre Chemetoff et réalisé en 1994 constitue à ce titre une véritable démonstration. Avant l'intervention, une situation de confrontation regrettable minorait l'intérêt du front de mer havrais : un cordon de galets parsemé de cabines de plage se trouvait directement accolé à un boulevard urbain très passant, derrière lequel la ville semblait privée de son rapport à la mer. L'attractivité de la plage, son accessibilité, son image étaient alors loin de révéler leur potentiel actuel. Dans un habile travail de déclinaison de l'interface villeplage, à la faveur de la redécouverte d'un ruisseau, Alexandre Chemetoff a su déployer, comme un soufflet d'accordéon, une succession d'espaces de transition, multipliant les usages et les expériences : un boulevard apaisé avec des trottoirs élargis facilite les traversées, un jardin en creux accompagnant une promenade abritée du vent trouve son assise au bord du ruisseau remis au jour, une dune vient parfaire cet abri et créer une transition avec une pelouse de loisirs (jeux, scènes temporaires, festivals) accueillant de manière saisonnière bars et restaurants. Adossée, une promenade haute assure la continuité littorale et donne à voir la mer de manière confortable. En contrebas, un secteur est dédié à toutes les commodités estivales de la plage (cabines, dispositifs de surveillance, jeux, douches...) créant comme un seuil avant de gagner le cordon de galets et la plage (pratique des loisirs balnéaires et nautiques). La poursuite de l'aménagement de la promenade et sa desserte par le tramway depuis décembre 2012 amplifient l'attrait de ce qui est devenu en quelques années l'espace 
public le plus apprécié du Havre : agrément pour l'habitant, heureuse surprise pour le visiteur, carte postale valorisante pour la cité portuaire.

Figure 4. La métamorphose ou l'invention de la plage du Havre, l'espace public le plus fréquenté de l'agglomération

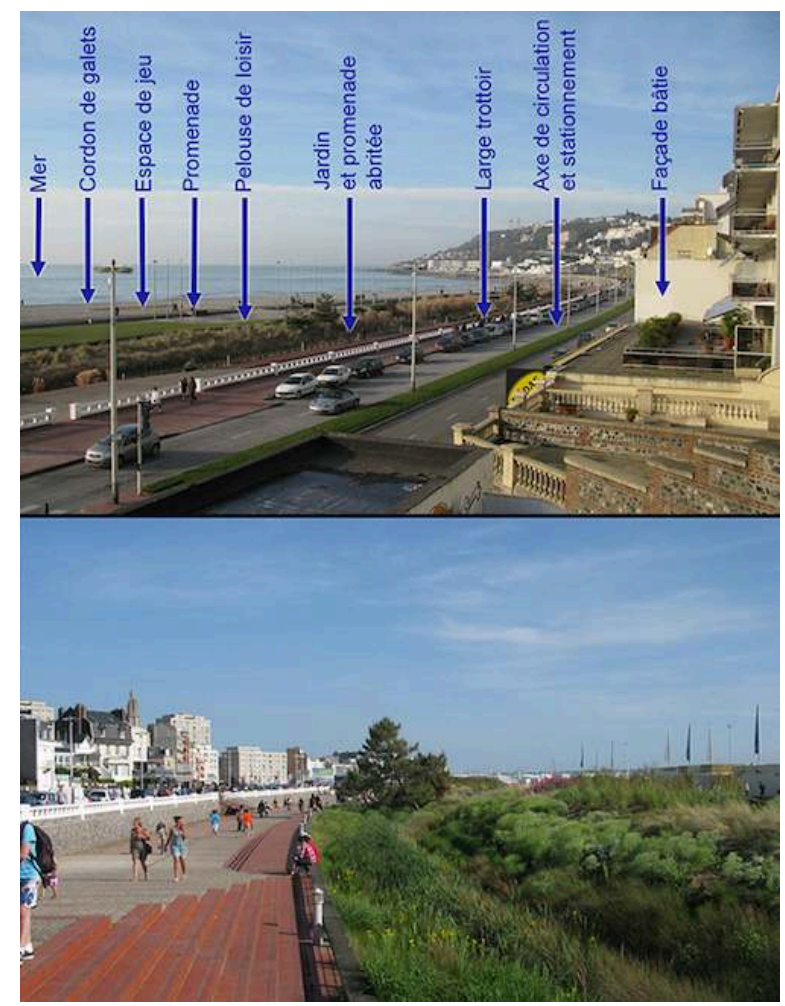

Projet d'Alexandre Chemetoff en 1994

Source : AURH (montage et photo).

L'autre référence majeure, incontournable pour les Seino-Marins, se trouve sur le plateau, elle constitue la structure paysagère identitaire qui ponctue le Pays de Caux. Ce vaste secteur agricole bénéficie de terres limoneuses particulièrement fertiles mais doit composer avec un environnement austère : forte exposition au vent, accès à l'eau potable, sols soumis aux phénomènes de battance et d'érosion. Son paysage «trouve son originalité dans la forme de son habitat rural, appelée "clos-masure", "courmasure" ou "cour". L'habitation et les dépendances agricoles se trouvent dispersées au sein d'une cour plantée de pommiers. L'ensemble est clos par un talus de terre, planté d'arbres de haut jet. Au caractère arboré et resserré des villages et des hameaux s'oppose l'étendue des plaines agricoles alentour constituées de champs ouverts. Cette organisation du territoire rural et les formes paysagères et bâties associées ne se rencontrent nulle part ailleurs que sur ce plateau crayeux, cerné par la Manche et la Seine. » (CAUE 76, 2008.) Ce fameux clos-masure apparaît comme une forme d'oasis au milieu de vastes plaines dominées aujourd'hui par les grandes cultures. Il articule l'échelle géographique du plateau avec celle de l'habitat rural et le cœur des exploitations agricoles. Atténuant les effets du vent, participant avec ses talus et fossés à la gestion des eaux pluviales, il domestique cet espace de plateau, lui donne des repères et des dimensions appréhendables et, par addition de clos dans le paysage 
cauchois, il fabrique des horizons boisés. Son organisation simple et remarquable à la fois constitue un réservoir de solutions pour gérer des interfaces en milieu rural.

Figure 5. Structure et photo aérienne du clos-masure

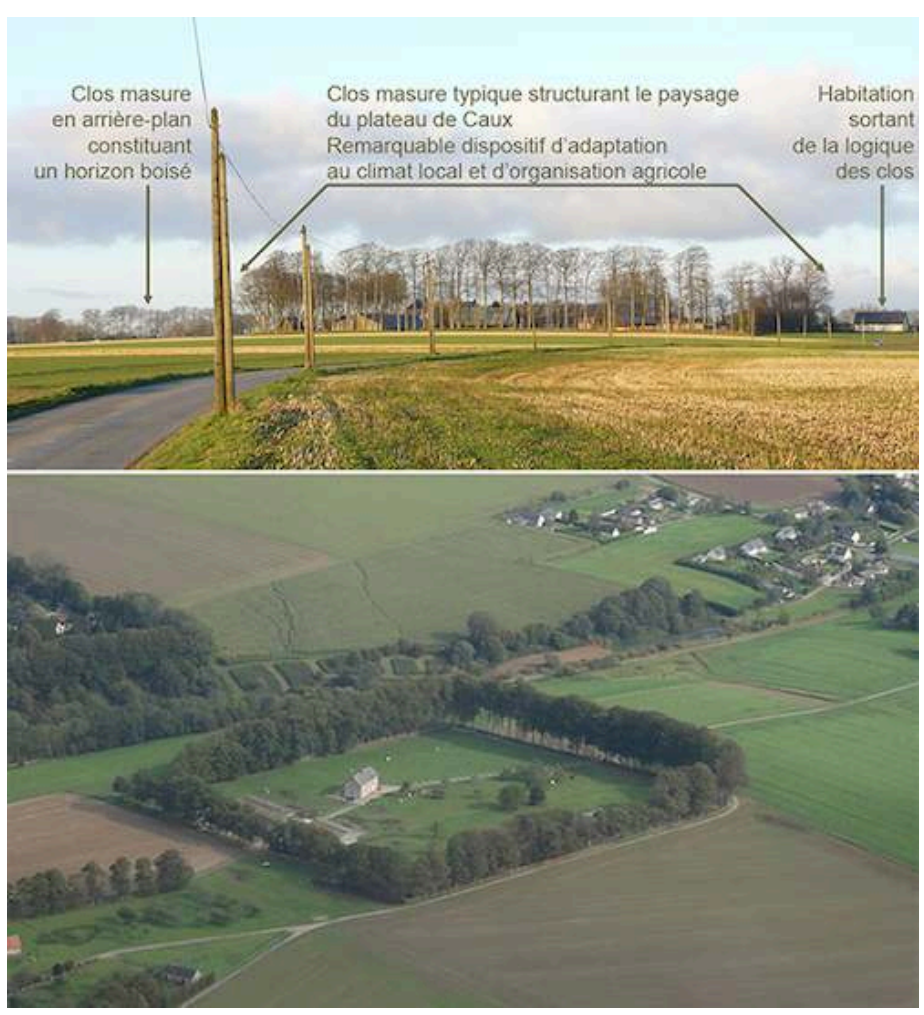

Photo du haut : la structure du clos-masure est rendue lisible par la relative transparence des haies d'arbres de haut-jet en hiver, la maison bâtie hors du clos à droite apparaît incongrue et sans accroche dans un paysage d'openfield; photo du bas : clos-masure ayant conservé sa vocation agricole.

Source : AURH (montage et photo aérienne)

\section{Coconstruire les propositions de lisières contemporaines de la Pointe de Caux}

Si l'idée d'un atelier concernant les lisières est rapidement apparue comme un sujet pertinent pour la valorisation de notre territoire, nous avons souhaité bien penser le processus en amont. En premier lieu, il devait tendre vers une dimension plus opérationnelle que celle de nos travaux précédents (Scot, charte, schéma directeur, projets urbains): il s'agissait d'élaborer des pistes de propositions concrètes en adéquation avec les réalités du terrain (configuration physique, enjeux locaux). Nous avons visé trois objectifs principaux dans le dispositif retenu: assurer une acculturation (enjeux de territoire, démarches paysagères), coconstruire des propositions de création ou d'amélioration de lisières et permettre une appropriation du travail par les principaux acteurs (élus, techniciens) susceptibles d'envisager sa mise en œuvre. Nous avons également identifié des enjeux qui justifiaient un travail sur les lisières: la gestion des eaux pluviales, l'adaptation au changement climatique, le développement économique et industriel, le cadre de vie, la biodiversité à travers le déploiement de la trame verte et bleue, l'agriculture... Enfin, il est important de 
constater que l'ampleur des mutations du territoire (dynamiques périurbaines, mutations agricoles, déploiement des activités industrialo-portuaires, enjeux métropolitains) constituait de fait une motivation à chercher des pistes nouvelles dans les processus d'aménagement du territoire tant dans une logique d'ancrage territorial que de développement de nouvelles aménités.

Nous avions résumé ainsi la spécificité de l'approche de cet atelier : priorité au terrain, pas de phase diagnostic et un processus dynamique. Ce laboratoire d'idées rassemblait cinq groupes complémentaires : le syndicat mixte, représentant administratif du Scot, à l'origine de la commande ; six élus référents, membres du Scot, assurant l'encadrement politique de l'atelier (comité de pilotage); l'École nationale supérieure du paysage, fournissant les forces vives de l'atelier, avec trois étudiants paysagistes et un encadrant professionnel paysagiste ; l'assistance assurée par l'AURH s'appuyant également sur son paysagiste pour gérer la préparation, l'organisation, l'animation et le suivi du dispositif pour le compte du syndicat mixte du Scot; et enfin, une quarantaine de techniciens représentant la diversité des domaines appréhendés dans l'atelier (comité technique). Plus d'une cinquantaine de personnes se sont trouvées ainsi régulièrement mobilisées sur cet atelier qui a connu sur le territoire une réunion de lancement, trois comités techniques et trois comités de pilotage entre octobre 2013 et mars 2014.

Après les premières visites de terrain et rencontres d'acteurs, le processus s'est enclenché, les étudiants ont commencé à formuler des propositions en utilisant tout leur précieux outillage de représentation graphique (photos, croquis, plans, coupes, perspectives cavalières). Les techniciens et les élus ont trouvé matière à débattre, ajuster et amender les propositions qui se sont précisées et étoffées au fur et à mesure de l'avancement de l'atelier. L'abondance, la pertinence et la qualité de la production ont rapidement donné du crédit aux trois jeunes paysagistes. Par leur créativité, ils ont démontré que les lisières constituent des espaces du possible. Ces dernières sont propices à l'innovation pour peu qu'on change la manière de les appréhender (un nouveau regard) et qu'on accepte le renouvellement de leurs formes. L'atelier a ainsi suscité l'inventivité du territoire et de ses acteurs, mobilisé l'intérêt collectif. 
Figure 6. Planche extraite de la publication liée à l'atelier montrant un exemple des outils graphiques mobilisés par les jeunes paysagistes pour traduire les propositions débattues avec les différents acteurs (cet extrait concerne le plateau agricole du territoire)
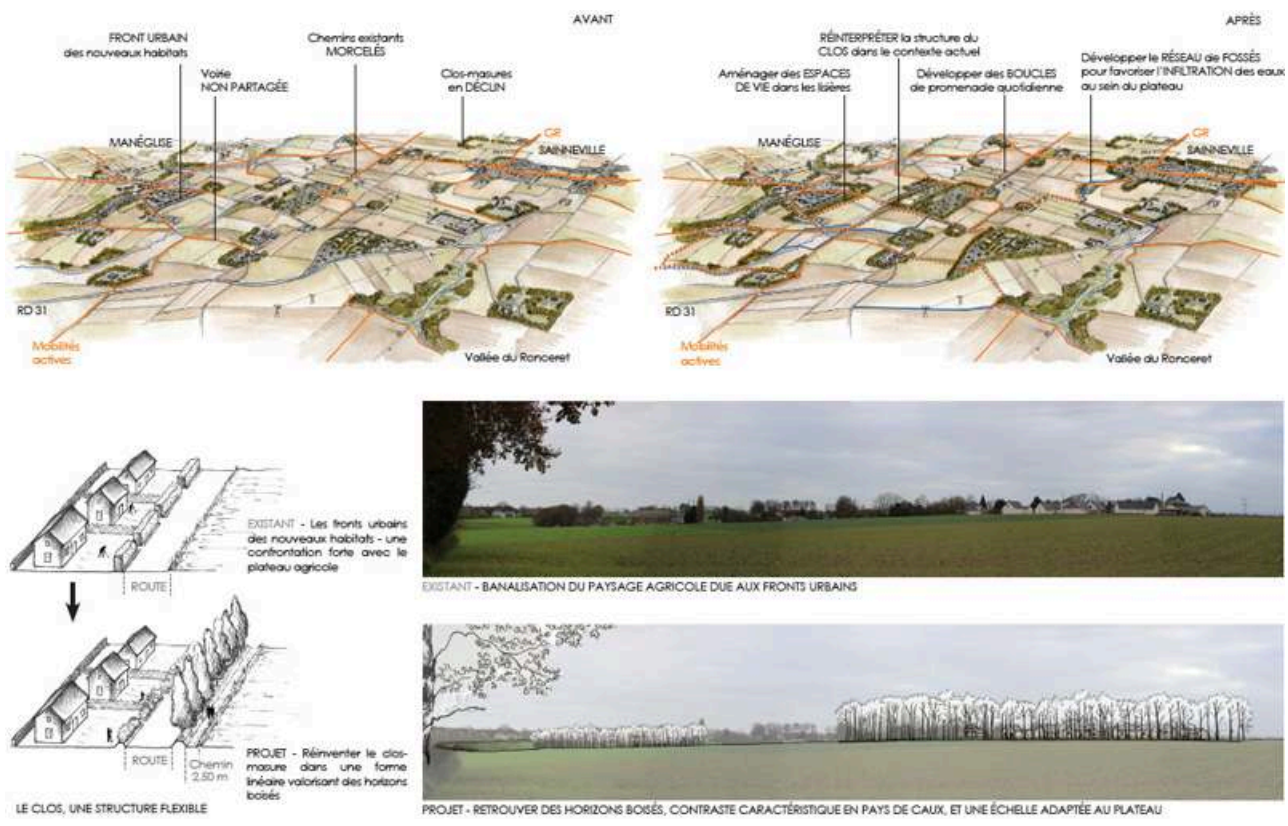

Les perspectives cavalières permettent de montrer le déploiement de la stratégie à une échelle territoriale. Les schémas de principe proposent des zooms sur les modalités d'organisation et les photomontages simulent les effets visuels attendus.

Nous avons constaté que ces espaces de lisière constituent les vitrines successives du territoire. Aussi leur évolution participe-t-elle de l'identité du territoire à venir, de son attractivité, questions fondamentales tant pour les élus, que pour les habitants et les acteurs économiques. La structuration des lisières, leur mise en projet peuvent constituer un moyen de lutte contre la banalisation, la perte de repères mais aussi permettre de développer de nouveaux espaces de vie, d'accueillir de nouvelles fonctions. C'est ce qui ressort de l'ensemble des propositions réunies dans l'ouvrage dédié Pour de nouvelles rencontres en Pays de Caux (Baboulène, Gouchault, Olivier, 2014).

\section{La frange urbaine nord du Havre : concevoir un «territoire lisières »}

19 Le plateau au Nord-Ouest du Havre accueille un secteur à dominante agricole aux marges des villes du Havre (au sud, moins de 175000 habitants en 2012), d'Octevillesur-Mer (au nord-ouest, environ 5800 habitants en 2012) et de Fontaine-la-Mallet (au nord-est, pratiquement 2700 habitants en 2012). Il constitue un lien avec le vaste plateau de Caux aux terres particulièrement fertiles, secteur d'intensification des pratiques agricoles aujourd'hui marqué par la prédominance des grandes cultures (blé, betterave, pomme de terre). Ce secteur d'entrée d'agglomération bénéficie d'atouts majeurs. À l'ouest, malgré la présence de l'aéroport, les falaises de la Côte d'Albâtre créent une vaste ouverture maritime (vue, microclimat, écologie particulière). À l'est, les 300 ha de la forêt de Montgeon proposent une offre de nature et de loisirs importante pour les habitants qui se prolonge par le parc de Rouelles (séquence de vallée de 160 ha dédiée aux usages récréatifs et gérée de manière différenciée) qui 
débouche dans la vallée structurante de la Lézarde. Le cœur du plateau nord-ouest est occupé par une agriculture diversifiée : des parcelles de grandes cultures appartenant à des grosses fermes du plateau côtoient un peu d'élevage bovin, une quinzaine d'exploitations maraîchères, des activités équestres, et des animaux en pâture correspondant à une petite agriculture de loisirs (moutons, chèvres, chevaux). Ces activités cohabitent dans un espace marqué par la présence d'habitat dispersé, d'ouvrages de gestion hydraulique et d'infrastructures d'échelle d'agglomération. La rocade nord, ouvrage routier conséquent, a créé récemment (inauguration en octobre 2012) une frontière physique entre Le Havre et le plateau agricole et constitue pour le moment une limite à l'urbanisation de la commune. Le tramway a positionné son terminus en décembre 2012 sur cette même limite. Il est intéressant de noter que parallèlement, comme un premier jalon d'intégration des travaux de concertation entre la sphère agricole et des représentants de culture plus urbaine (2008 à 2010), ce secteur avait pu rentrer dans le giron des trois espaces dits d'« enjeux agricoles partagés » du Scot approuvé en 2012. Ce statut vise à garantir, malgré la forte pression propre à cette position périurbaine convoitée par bien des projets d'extension (bâtis, zones d'activité, infrastructures), une vocation agricole à moyen ou long terme.

Figure 7. Extrait de la carte du projet agricole du Scot approuvé en février 2012 (document d'orientations générales du Scot LHPCE)

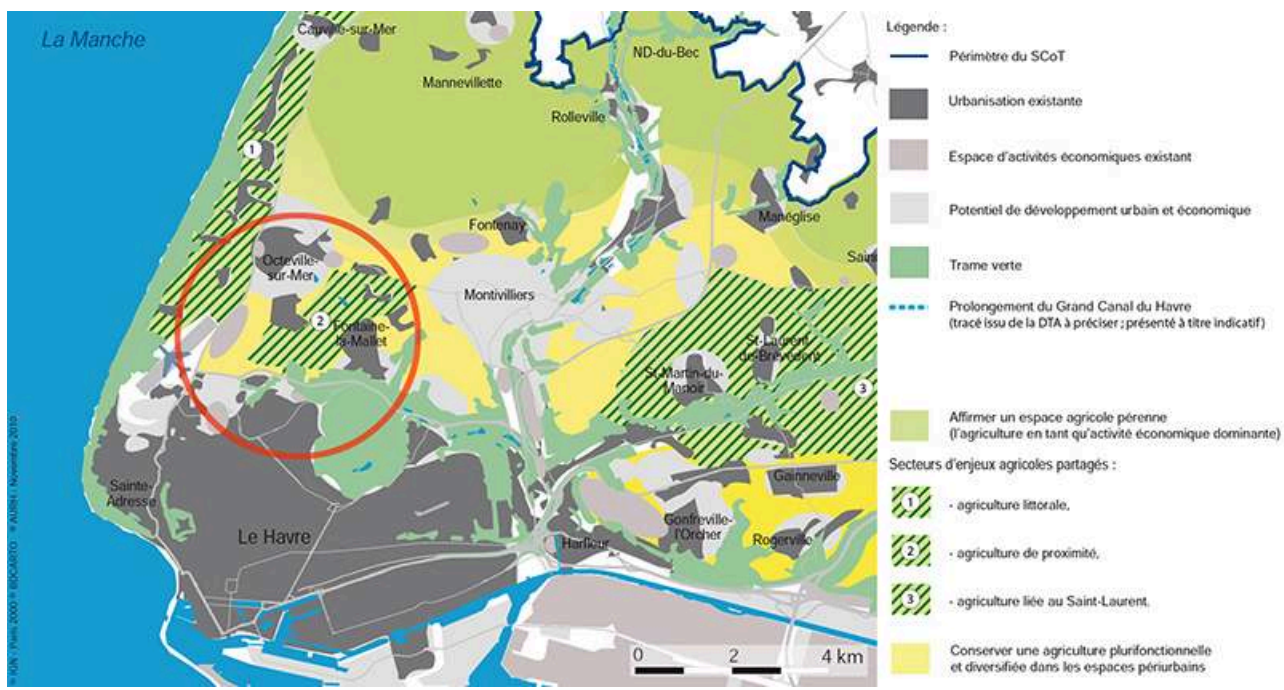

Les secteurs hachurés correspondent à des ensembles fonciers que les élus ont souhaité voir maintenus dans leur vocation agricole malgré la forte pression urbaine dont ils font l'objet. Le secteur 1 caractérise une agriculture dite littorale tandis que le secteur 2 est plus clairement lié à une agriculture dite de proximité. Ces deux secteurs participent de la diversité du plateau nord-ouest du Havre et y garantissent une composante agricole forte à l'horizon du Scot. Le cercle rouge identifie l'espace périurbain dont il est plus précisément question dans l'article.

Pour un urbaniste ou un paysagiste, ce secteur porte à la fois les signes d'une situation d'équilibre précaire qui pourrait préfigurer une urbanisation latente (morcellement du parcellaire agricole, activités économiques peu qualifiantes, rocade pouvant offrir de nouvelles dessertes efficaces) mais bénéficie également de tous les ingrédients attendus pour y créer un espace unique en accord avec les attentes citadines (agriculture de proximité, loisirs verts, cheminements, massif forestier, parc, bordure littorale...). Cet état intermédiaire se traduit dans le paysage par des situations d'attentes (lotissement inachevé, branches de rond-point non fonctionnelles, talus temporaires) qui côtoient 
une dynamique de projets d'un genre nouveau (cueillette, nouveaux cheminements doux, bassins d'orage pensés en réseau pour la biodiversité et l'agrément des promeneurs).

Dans ce secteur fragmenté, la question des lisières se démultiplie en fonction de la diversité des usages des sols rencontrés, de leurs évolutions probables mais aussi en fonction de l'échelle d'appréhension de celles-ci. Malgré quelques tracés historiques encore lisibles, il règne une forme de confusion quand on s'écarte d'espaces aussi structurants que la forêt, le cœur villageois d'Octeville-sur-Mer ou la falaise. Aussi, l'atelier n'a-t-il pu se contenter de travailler sur des lisières ponctuelles, il s'est trouvé devant la nécessité de défendre un plan d'action multiscalaire. En effet, nous avons dû considérer l'ensemble territorial interrogé comme une vaste lisière à traiter pour en proposer un tout cohérent. Sans que le débat ne soit vraiment tranché, il est apparu nécessaire de qualifier ce vaste ensemble, de le mettre en perspective avec une idée de projet structurant en filigrane, un parc agricole d'agglomération, intuition restant à étayer. Alors en s'appuyant sur la nécessité de maintenir des espaces agricoles cohérents et connectés avec le plateau de Caux, en se servant des vallées et talwegs comme supports d'un maillage multifonctionnel (trame verte et bleue, réseau piétonsvélos, interfaces paysagères de grande ampleur, gestion des eaux pluviales), en proposant un réseau de déplacements de proximité piétons-vélos-chevaux à cet ensemble, un projet a pris forme. En redonnant du sens à ce secteur, nous avons orienté l'élaboration des réponses fonctionnelles et formelles. Les étudiants ont proposé des " portes » d'accès à cet espace, ils ont retravaillé les fronts urbains hasardeux avec des logiques de transition, ils ont formulé des pistes pour gommer les effets de frontière (rocade nord) et reconnecter le territoire à son espace maritime. Les interventions proposées ont dessiné par addition une nature intermédiaire, un espace nourricier et accueillant. Une meilleure manière de mutualiser des fonctions a été envisagée (exemple : rétention d'eau et usages de loisirs). Ainsi, l'agriculture pourrait-elle gagner les espaces verts urbains et les cheminements des parcs et jardins se prolonger dans des «morceaux de campagne réinventés». Dans cette configuration, un espace agronaturel à vocation plus urbaine se constituerait, intermédiaire entre la ville et un plateau orienté vers l'agriculture intensive, un lieu majorant les capacités de résilience de l'agglomération. Cet espace nécessiterait un positionnement intercommunal car il prendrait tout son sens en étant pensé comme un cœur plus que les périphéries ajoutées du Havre, d'Octeville-sur-Mer et de Fontaine-la-Mallet. À l'échelle du Scot, le traitement proposé ferait du plateau nord-ouest un lieu ressources, garantirait les continuités des grandes trames structurantes (écologique, hydraulique, mobilités douces...), un véritable territoire lisières. 
Figure 8. Extrait de l'atelier (planche recomposée)

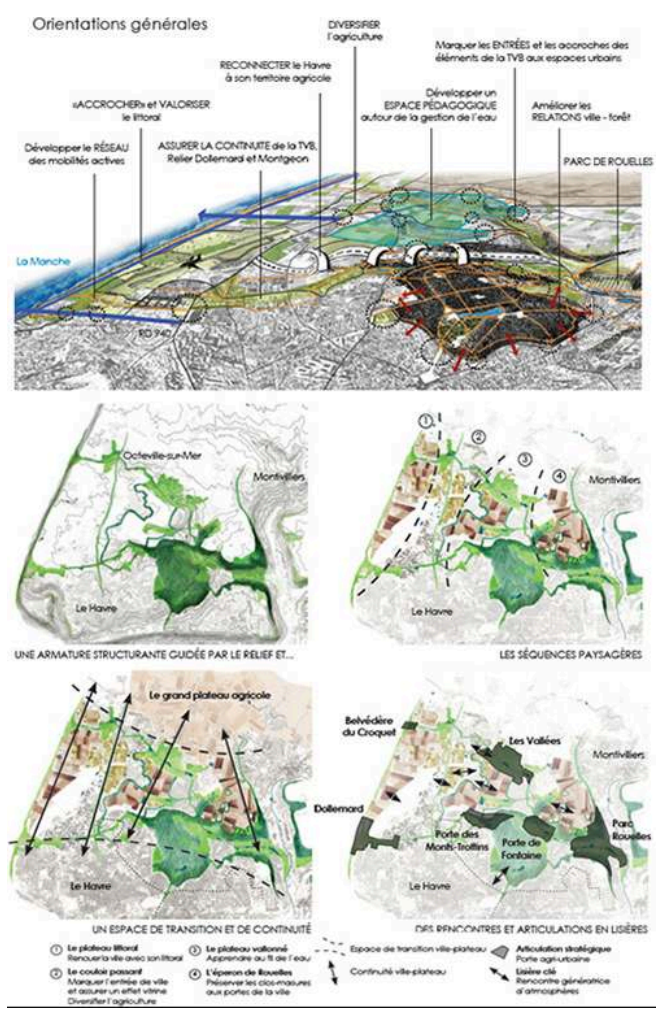

Une approche à l'échelle du territoire rendue nécessaire pour proposer des réponses pertinentes dans ce secteur dont la complexité réside dans la diversité des dynamiques à l'œuvre.

Figure 9. Extrait de l'atelier (planche recomposée)

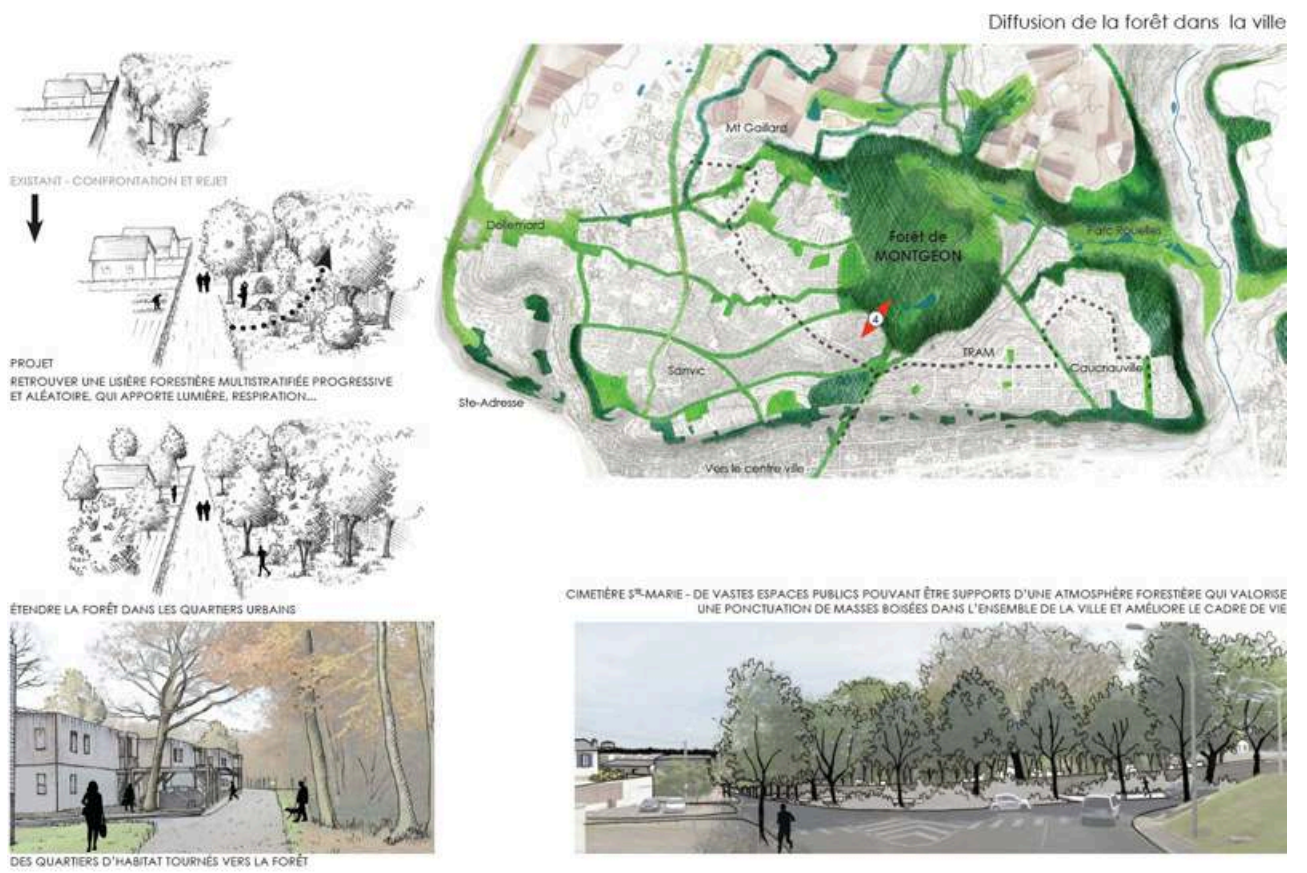

Comment diffuser l'ambiance forestière au sein de la ville et créer des hybridations entre espaces urbains et forestiers plutôt que des ruptures franches. Quelques exemples de propositions. 


\section{Un territoire réaffirmé par un « canevas » porteur de sens}

22 Ancrées dans les problématiques du territoire, débattues avec les acteurs clés, les différentes pistes d'action de cet atelier constituent un recueil de projets qui reste à mettre en œuvre. En ouvrant le champ des possibles, ce recueil a pour objectif d'interpeller et de déclencher des initiatives. Les premières ont d'ailleurs eu lieu dans les lisières de la forêt de Montgeon l'hiver 2014-2015.

La somme de toutes ces propositions complémentaires donne au projet une portée à l'échelle du Scot. C'est un canevas porteur de sens dont la concrétisation permettrait d'affirmer un cadre paysager contemporain attractif. Ce canevas accompagnerait les dynamiques de transformation tout en installant de nouveaux repères durables, des échelles intermédiaires propices à accueillir des lieux multifonctionnels et des usages collectifs attendus par les habitants. Les espaces de transition constituent de véritables laboratoires qui appellent diversité de pratiques et innovation. Au Havre, l'AURH travaille à ce titre activement sur l'interface ville-port qui regorge de possibilités de diversification économique, de potentiel de renouvellement des formes urbaines et industrielles, d'invention de nouveaux usages. Comme le travail le montre, la limite devient généralement intéressante et fertile quand elle offre une certaine épaisseur et qu'elle devient un possible espace de partage.

Figure 10. Extrait de l'atelier (planche recomposée)

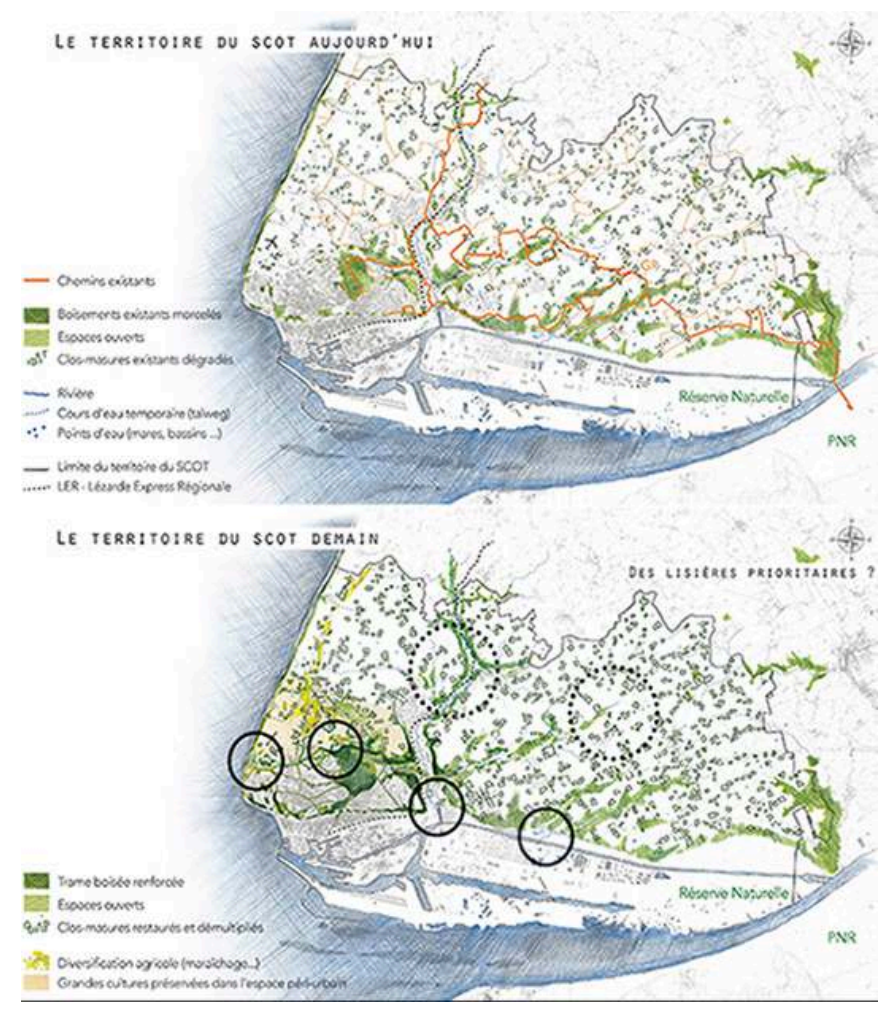

Les bénéfices à l'échelle du Scot. Une série de cartes décline les effets à cette échelle : renforcement de la trame verte et bleue, valorisation du rapport à l'eau, développement à l'échelle du territoire du maillage de cheminements doux, valorisation de points de vue... La dernière carte ci-dessus propose quant à elle d'orienter un plan d'actions en débutant par des lisières prioritaires qu'elle identifie (cercles en plein et en pointillé). 
Le travail réalisé dans le cadre de cet atelier constitue une base commune de réflexion itérative à partir de laquelle les acteurs peuvent continuer à développer de nouvelles réponses avec une logique interdisciplinaire. Il vient servir l'élaboration du nouveau Scot (horizon d'approbation 2017-2018) et enrichir la charte paysagère et environnementale qui l'accompagne. Certaines propositions peuvent être anticipées par leur intégration au sein des outils de planification tels que les PLU. Il faut rappeler que cet atelier a constitué une belle opportunité pour les élus et les techniciens locaux d'accueillir un regard extérieur dynamisant sur leur territoire. Il a remis en lumière et en question des structures paysagères, des projets et des lieux clés. Les propositions pourront agir également comme des leviers pour débloquer des situations complexes par la voie du projet. La frange urbaine nord du Havre apparaît comme le secteur de projet où un approfondissement du travail s'avérerait sans doute le plus profitable tant les premières propositions ont créé des perspectives d'améliorations notables.

Les premiers remerciements vont à Céline Baboulène, Marlène Gouchault et Étienne Olivier, forces vives de cet atelier, dont l'enthousiasme, les capacités d'échange et d'intégration ainsi que l'investissement considérable dont ils ont fait preuve ont conduit à la réalisation d'un travail riche et passionnant pour l'ensemble des interlocuteurs concernés. Ils ont également su se mobiliser à trois reprises pour venir m'accompagner dans des présentations de ce travail au-delà du cadre de l'atelier.

Il faut remercier de manière au moins comparable Bertrand Folléa qui a su guider cette énergie créative sans jamais perdre de vue les objectifs premiers. Il a habilement poussé les étudiants à développer une stratégie pour que l'addition de leurs propositions prenne tournure à l'échelle du Scot. Il a toujours été un pédagogue bienveillant, redoutablement précis et efficace.

Le travail de préparation de cet atelier a été affiné avec le CAUE 76 dont le directeur, Olivier Gosselin, et la paysagiste, Virginie Maury-Deleu, associés de longue date aux travaux du Scot, partagent cet intérêt pour les espaces de l'entre-deux.

Naturellement les six élus du Scot ainsi que la quarantaine de techniciens méritent également d'être chaleureusement remerciés pour leur active participation faite de contributions, de critiques constructives et d'encouragements qui ont permis de garantir la justesse des propositions.

Enfin, l'équipe de l'AURH doit être citée pour son investissement, en particulier Jessy Oukoloff, chef de projet du Scot, Alix Guillemette, chargée d'étude développement durable. Alix Gay, chargée de communication, a su accompagner habilement les processus de valorisation des travaux au travers de leur publication, des retours presse et des encarts sur le site Internet de l'AURH. 


\section{BIBLIOGRAPHIE}

Les Atlas de paysages, méthode pour l'identification, la caractérisation et la qualification des paysages, Paris, ministère de l'Écologie, du Développement durable et de l'Énergie, direction générale de l'Aménagement, du Logement et de la Nature, Paris, 2015, fiche technique nº 1, p. 64.

Baboulène, C., Gouchault, M., Olivier, E., Pour de nouvelles rencontres en Pays de Caux, Le Havre, AURH, syndicat mixte du Scot LHPCE, 2014.

Beltrando, Y., Lecroart, P., Palisse, J.-P.., « Initier des projets métropolitains : l'IBA, une pratique féconde », Paris, Note rapide, $\mathrm{n}^{\circ}$ 478, Apur, 2009.

Conseil d'architecture d'urbanisme et d'environnement de Seine-Maritime, Clos-masures et paysage cauchois, Évreux, Éditions Point de Vue, 2008.

Donadieu, P., Campagnes urbaines, Arles/Versailles, Actes Sud/ENSP, 1998.

Mangin, D., La Ville franchisée, Paris, Les Édition de La Villette, 2004.

Masboungi, A. (dir), Le Paysage en préalable, Michel Desvigne. Grand Prix de l'urbanisme 2011, Marseille, Éditions Parenthèses, 2011.

Menguy, B., Guibert, V., Vers une plus grande attractivité, Le Havre, AURH, 2014.

Reichen, B., Peter, A. \& associés, « Scot de l'agglomération de Montpellier », Montpellier agglomération, 2006.

Tachon, C.-H., « Mon village en l'an 2000 », Le Visiteur, n 3, Paris, Société française des architectes, 1997, p. 6-35.

Thiberghien, G., Corner, J., Natures intermédiaires, Bâle, Birkhäuser, 2009.

Urbanisme. Urbain/Rural, n 338, Paris, 2004.

Urbanisme. Campagnes urbaines, $34^{e}$ rencontre des agences d'urbanisme, hors série n 47, Paris, 2013.

\section{NOTES}

1. En 2009, les 10 équipes d'architectes chargées de proposer leur vision de la future agglomération parisienne présentent leurs projets. L'équipe emmenée par Antoine Grumbach met en avant Le Havre comme port de Paris et plaide pour un vaste projet métropolitain, d'échelle Vallée de la Seine, "Seine métropole». Cette vision sera soutenue par le président Sarkozy, notamment dans son discours au Havre en juillet 2009 «Faire du Grand Paris une véritable métropole maritime ".

2. L'AURH œuvre à la connaissance et à l'aménagement du territoire dit de l'Estuaire. Ce territoire politique couvrant 5 Scot se constitue en tant que façade maritime du projet de la Vallée de la Seine. Observation, prospective, planification, projets urbains..., les missions sont diverses et complémentaires.

3. En 2008, le premier Scot LHPCE était approuvé mais avec un souhait des élus de l'enrichir rapidement sur les questions de paysage, d'environnement et d'agriculture. Pour répondre à leurs attentes, l'AURH a mené un travail d'étude et de propositions qui a trouvé plusieurs formes d'aboutissement: l'élaboration d'une charte paysagère et environnementale, la mise en ligne d'un espace Internet dédié, l'enrichissement du Scot approuvé en février 2012 et enfin la publication de l'ouvrage Vers un plus grande attractivité (Menguy et Guibert, 2014). 
4. La charte paysagère et environnementale du Scot LHPCE est guidée par les engagements des élus approuvés en 2011. Elle constitue une démarche vivante basée sur le volontariat en complément du Scot (cadre réglementaire). Sa dynamique d'animation (conseils, ateliers, visites d'opération de référence...) et de capitalisation d'expériences met à disposition des élus et des techniciens du territoire des références en matière d'aménagement durable en lien avec leurs attentes.

5. L'agence d'urbanisme a précédemment contribué à deux autres ateliers pédagogiques régionaux avec l'ENSP. Le Grand Port maritime du Havre, commanditaire, a souhaité s'interroger sur son image, sa mise en scène et les leviers possibles d'évolution de certains secteurs stratégiques. Ces deux ateliers ont permis d'explorer respectivement, en 2012 puis en 2013, la place de la Zone industrialo-portuaire au sein de la plaine alluviale de la Seine. La question des limites et des lisières a été évoquée et travaillée de sorte qu'il n'a pas été utile que l'atelier du syndicat mixte du Scot LHPCE se penche de nouveau sur les secteurs ayant déjà fait l'objet de réflexions et de propositions.

6. Les structures paysagères désignent les systèmes formés par les éléments de paysage. Les interrelations entre ces éléments peuvent être matérielles ou immatérielles, supportées par des liens fonctionnels, topographiques ou symboliques. Les structures paysagères constituent les traits caractéristiques d'un paysage. Elles revêtent une grande importance, car c'est sur elles que porte l'action publique (Les Atlas de paysages, 2015, p. 64).

\section{RÉSUMÉS}

Si la planification parvient à rendre lisibles des intentions politiques et à nous préserver de certains excès, elle peine toutefois à nous prémunir des fractures issues du zonage et des découpages fonciers. Les limites administratives ne font pas souvent sens et se révèlent inaptes à favoriser des usages fructueux de l'espace ou à garantir une qualité de paysage. En 2014, un atelier initié par l'Agence d'urbanisme de la région du Havre et de l'Estuaire de la Seine (AURH) s'est mobilisé autour de cette problématique et a permis de mettre au point, grâce au précieux concours de trois élèves de $4^{\mathrm{e}}$ année de l'École nationale supérieure du paysage (ENSP), une centaine de propositions de lisières inédites sur cinq entités paysagères du schéma de cohérence territoriale Le Havre Pointe de Caux Estuaire (Scot LHPCE). L'article revient sur les attentes d'attractivité du territoire, la démarche de coconstruction des propositions et se focalise sur les questions soulevées par la frange nord-ouest du Havre, secteur périurbain clé dont la démarche vient réinterroger le devenir. La qualification de lisières peut-elle véritablement définir de nouveaux espaces et accompagner des changements de pratiques?

Although planning makes it possible to express political intentions and provide safeguards against certain excesses, it does sometimes fail to protect us from divisions caused by land zoning. Administrative limits often make no sense and are inappropriate for ensuring profitable use of land space or guaranteeing the quality of the landscape. In 2014, a workshop organised by the urban planning agency of the region of Le Havre and the Seine estuary area focused on this question and made it possible to develop, with the valuable contributions of three fourth year students from the Ecole national supérieur du paysage (ENSP), approximately one hundred original proposals for fringe areas belonging to five landscape entities included in the master urban plan for the Le Havre Pointe de Caux estuary area. The article refers to expectations in terms of the 
attractiveness of the area, describes the co-design approach for generating the proposals and focuses on the questions raised by the north-western fringe of the Le Havre, a key suburban area the development of which presents new prospects for the future. Can redesigning these fringe areas contribute to defining new spaces and developing new practises?

INDEX

Mots-clés : lisière, aménités, rencontre, multifonctionnalité, hybridation

Keywords : fringes, amenities, community life, multi-functionality, hybridation

\section{AUTEUR}

\section{BORIS MENGUY}

Paysagiste DPLG, Boris Menguy est chef de projet ingénierie du développement durable à l'Agence d'urbanisme de la région du Havre et de l'Estuaire de la Seine (AURH).

b.menguy[at]aur[dot]fr 